\title{
Should Fatigue Be Considered as A Long-Term Sequela After Sepsis?
}

\author{
Gundula Seidel ${ }^{1,2 *}$, Theresa Götz ${ }^{3}$ and Farsin Hamzei ${ }^{1,2}$ \\ ${ }^{1}$ Department of Neurology, Moritz Klinik Bad Klosterlausnitz, Germany \\ ${ }^{2}$ Department of Neurology, Section of Neurological Rehabilitation, Jena University Hospital, Germany \\ ${ }^{3}$ Department of Computer and Data Sciences, Jena University Hospital, Germany
}

Submission: December 04, 2018; Published: March 13, 2019

*Corresponding author: Gundula Seidel, Moritz Klinik, Herrmann-Sachse-Str 46, 07639 Bad Klosterlausnitz, Germany

Abstract

Objective: Cognitive long-term impairment is a relevant sequela after sepsis. Certain diseases accompanied with cognitive decline are often also associated with fatigue (e.g. multiple sclerosis). The aim of this pilot report is to investigate if sepsis survivors with persistent cognitive deficits also suffer from fatigue syndrome.

Methods: In this cross-sectional study, 24 survivors of severe sepsis with persistent cognitive deficits were being examined by means of a neuropsychological test battery and interviewed on the basis of two German fatigue scales.

Result: 22 of the 24 sepsis survivors (91.7\%) reported relevant fatigue symptoms. Considering their most relevant impairment of cognitive domain (alertness), sepsis survivors showed a close relationship between alertness and fatigue (higher fatigue scores were associated with lower alertness performance).

Conclusion: Fatigue is also a long-term sequela of sepsis survivors with persistent cognitive decline. As a result, this should be considered in rehabilitation of sepsis survivors, e.g. by a specific fatigue treatment and / or a training of alertness. In addition, this also raises the question whether sepsis survivors without cognitive impairment also suffer from fatigue syndromes, which could be another promising field of research.

Keywords: Sepsis, Fatigue, Cognitive outcome, Rehabilitation

Abbrevations: WEIMuS: Würzburger Erschöpfungsinventar; FSMK: Fatigue Skala für Motorik and Kognition; ICU: Intensive Care Unit

\section{Introduction}

Sepsis survivors have an increased risk for a long-term cognitive impairment which could affect various key domains of neuropsychological functioning, e.g. attention, memory or executive functioning [1,2]. Cognitive decline has been also detected in connection with certain diseases associated with fatigue (e.g. multiple sclerosis or stroke). In this regard, fatigue is defined as an overwhelming sense of tiredness, lack of energy or feeling of exhaustion [3]. A systematical investigation if fatigue is also a relevant long-term outcome in sepsis survivors suffering from persistent cognitive deficits has not been conducted so far. Therefore, the aim of this pilot observational study was to analyze if sepsis survivors with cognitive impairment also suffer from fatigue.

\section{Participants and Methods}

The inclusion criteria were a sepsis or septic shock episode with subsequent cognitive impairment dating back to at least more than one year. Sepsis and septic shock were defined according to the sepsis-3 consensus [4]. To verify cognitive impairment, participants were tested with a comprehensive neuropsychological battery targeting different domains of cognition: alertness, divided attention, selective attention, working memory, memory span, learning capacity, delayed recall and rate of decay, visuo-spatial ability, and executive function. Participants were defined as cognitively impaired if they reached a performance below one standard deviation at 


\section{Open Access Journal of Neurology \& Neurosurgery}

least in two cognitive domains a or if they had a performance below 1.5 standard deviation at least in one domain.

Exclusion criteria were history of other neurological diseases (e.g. brain trauma, stroke, parkinson or multiple sclerosis) which alter the brain. Psychiatric symptoms (e. g. depression or schizophrenia). Further distinctions with regard to participants selection and characteristics are listed in Figure 1.24 participants whose sepsis or septic shock with subsequent persisting cognitive impairment dates back to more than one year were included (12 male, age $53.8 \pm 10.6$, years post sepsis $2.6 \pm 1.9$ ). All participants gave their written informed consent before participating in the study. This study was approved by the local ethics committee.

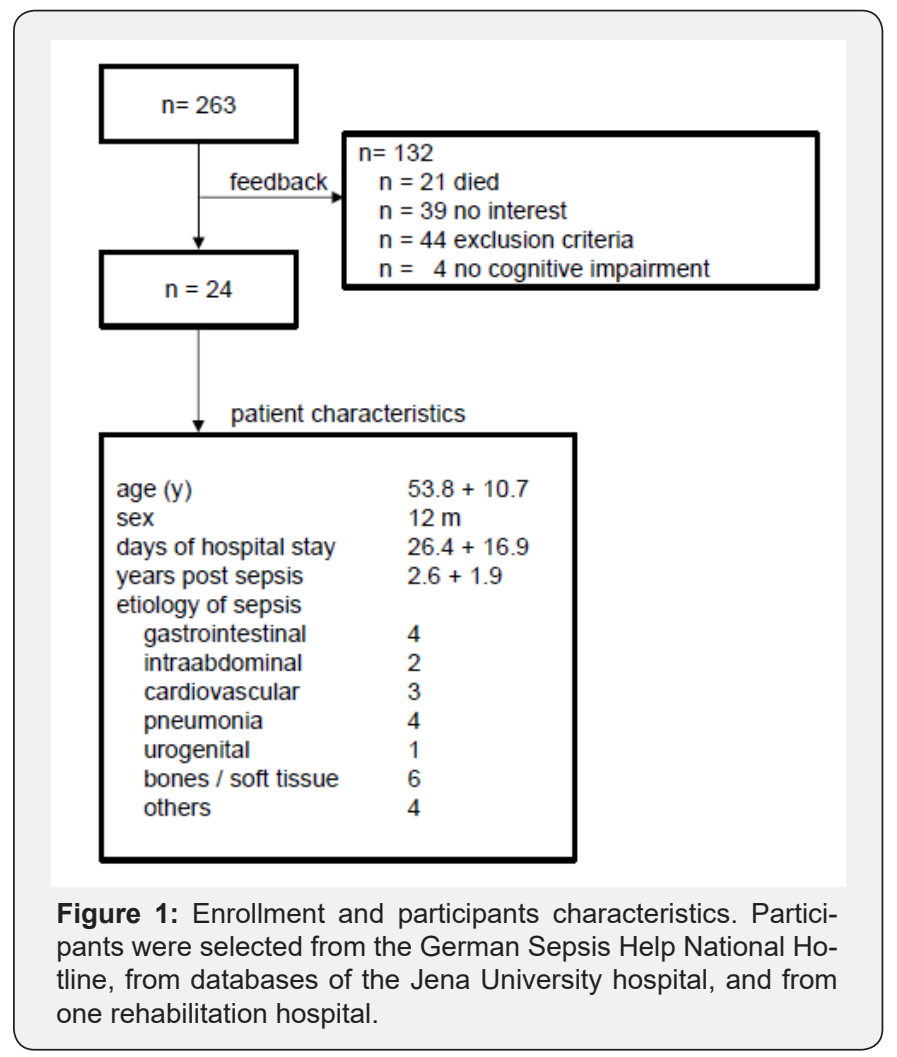

For fatigue assessment, two German fatigue questionnaires were used ("Würzburger Erschöpfungsinventar" WEIMuS [5] and "Fatigue Skala für Motorik und Kognition" FSMK [6], both contain items for cognitive and motor fatigue symptoms and corresponding cut-off values. Analyzing the relation between cognitive impairment and fatigue, first for both WEI MuS and FSMK, the scores were transformed into z-values and separately averaged for Fatigue total, Fatigue cognitive and Fatigue motor. The averaged z-values for fatigue were correlated with the T-values of those cognitive domains that were affected in more than $50 \%$ of the included participants. The significance threshold was set at $\mathrm{p} \leq 0.05$ (one-tailed, corrected for multiple comparisons according to Bonferroni-Holm).

\section{Results}

Tonic alertness was most frequently affected (in 13 participants; 54\%), followed by working memory (in 11 participants; 46\%), Memory functions were impaired in eight participants (33\%) regarding their learning capacity; seven participants $(30 \%)$ showed deficits in delayed recall, six participants $(25 \%)$ in memory decay rate, and two participants (8\%) in memory span. A total of 22 out of 24 participants $(91.7 \%)$ reached Fatigue total scores in WEIMuS or FSMK which exceeded the cut-off. For WEIMuS, the mean value of Fatigue total score came to $43.0( \pm 176.2 \mathrm{SD})$, the result for the sub score Fatigue cognitive was 21.7 ( $\pm 10.0 \mathrm{SD}$ ) and the sub score Fatigue motor amounted to 21.4 ( $\pm 7.1 \mathrm{SD})$. For FSMK, the mean value of Fatigue total score came to 74.8 ( $\pm 15.1 \mathrm{SD}$ ), the sub score Fatigue cognitive came to $36.5( \pm 9.0 \mathrm{SD})$ and the sub score Fatigue motor came to 38.3 ( $\pm 6.9 \mathrm{SD}$ ). Thus, fatigue (i.e. the averaged z-values of Fatigue total of WEIMUS and FSMK) significantly correlates with tonic alertness (higher alertness deficit correlated with more fatigue; (Figure 2a).

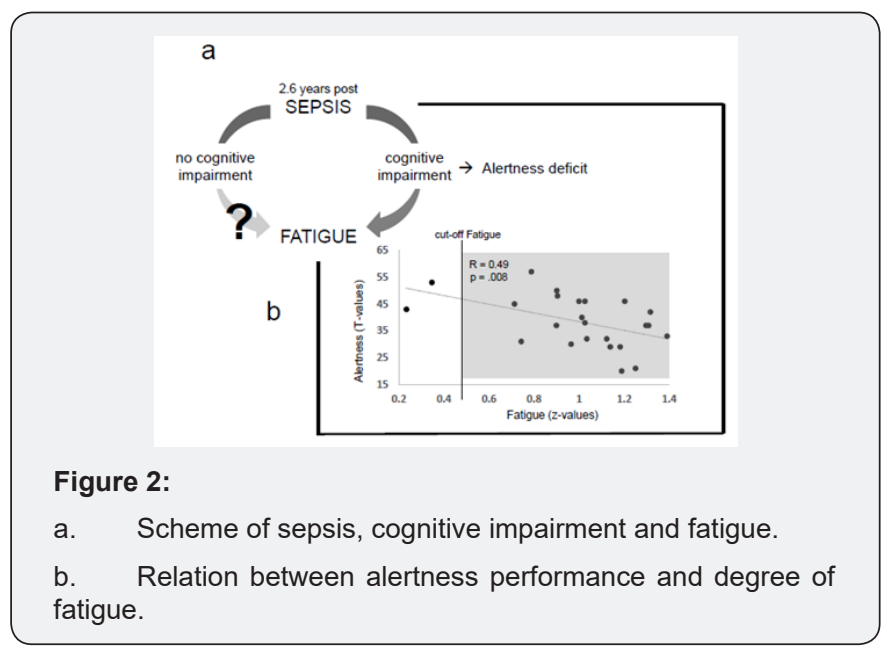

\section{Discussion}

Our new approach is to describe fatigue as a long-term sequela in our study population of sepsis survivors with cognitive deficits. In this respect, we found a close association between fatigue and alertness deficits.

Alertness is supposed to be the most basic aspect of attention providing higher attention functions as well as higher cognitive demands [7]. With a deficit in this domain, the basic attention functions are in turn being reduced, which consequently affects higher cognitive processes. Thus, with regard to cognitive impairment sepsis survivors are forced to deploy considerably more cognitive effort in order to solve complex tasks. Therefore, sepsis survivors reported symptoms of fatigue. This suggestion links the manifestation of fatigue to the alertness deficit. On the other hand, sepsis is a systemic inflammatory process that develops fatigue similar to other inflammatory diseases (multiple sclerosis, rheumatism) [8]. Such a depiction would link an inflammation process to a development of fatigue.

In our report we did neither consider clinical parameters during the ICU stay (e.g. delirium) [2] nor psychological (e. g. depression and anxiety) and personality variable (e.g. resilience). The lack of this data thus requires further longitudinal studies 
to verify factors that encourage fatigue after sepsis (Figure 2b). Furthermore, we cannot give a general assessment about the prevalence of fatigue in sepsis survivors because we only investigated sepsis survivors with cognitive impairment.

\section{Conclusion}

Fatigue must be considered in sepsis survivors. To refine the intervention, specific treatments like cognitive behavioral therapy or graded exercise therapy should be taken into account during rehabilitation. Moreover, targeted training of alertness may also improve fatigue symptoms and should be considered as a constituent part in rehabilitation of sepsis participants.

\section{Acknowledgement}

The authors would like to thank all the participants who have made the study possible through their support. The authors would like to thank Sebastian Bähr for proofreading and linguistic correction of the manuscript.

\section{Conflict of Interest}

The authors declare no competing financial interests.

\section{Funding}

The study was supported by the German Center for Sepsis Control \& Care (CSCC), funded by the Ministry of Education and Research (BMBF), grant no. 01 E0 1002.

\section{References}

1. Iwashyna TJ, Ely EW, Smith DM, Langa KM (2010) Long-term cognitive impairment and functional disability among survivors of severe sepsis. Jama 304(16): 1787-1794.

2. Pandharipande PP, Girard TD, Jackson JC, Morandi A, Thompson JL, et al. (2013) Long-term cognitive impairment after critical illness. N Engl j med 369(14): 1306-1316.

3. Krupp LB, Pollina DA (1996) Mechanisms and management of fatigue in progressive neurological disorders. Curr opin neurology 9(6): 456460.

4. Singer M, Deutschman CS, Seymour CW, Shankar-Hari M, Annane D, Bauer M, et al. (2016) The Third International Consensus Definitions for Sepsis and Septic Shock (Sepsis-3). Jama 315(8): 801-10.

5. Flachenecker P, Muller G, Konig H, Meissner H, Toyka KV, et al. (2006) Fatigue in multiple sclerosis. Development and and validation of the Wurzburger Fatigue Inventory for MS. Nervenarzt 77(2): 165-166, 168-170.

6. Penner IK, Raselli C, Stocklin M, Opwis K, Kappos L, et al. (2009) The Fatigue Scale for Motor and Cognitive Functions (FSMC): validation of a new instrument to assess multiple sclerosis-related fatigue. Mult scler 15(12): 1509-1517.

7. Posner MI, Petersen SE (1990) The attention system of the human brain. Annual review of neuroscience. 13: 25-42.

8. Streck EL, Comim CM, Barichello T, Quevedo J (2008) The septic brain. Neurochemical research 33(11): 2171-2177.

\section{Your next submission with Juniper Publishers will reach you the below assets}

- Quality Editorial service

- Swift Peer Review

- Reprints availability

- E-prints Service

- Manuscript Podcast for convenient understanding

- Global attainment for your research

- Manuscript accessibility in different formats ( Pdf, E-pub, Full Text, Audio)

- Unceasing customer service

Track the below URL for one-step submission https://juniperpublishers.com/online-submission.php 